\title{
RETROGRADE PERFUSION THROUGH THE SUPERIOR VENA CAVA PERFUSES THE BRAIN IN HUMAN BEINGS
}

\author{
Domenico Pagano, FRCS, ${ }^{\mathrm{a}}$ Chris M. Boivin, MPhil, ${ }^{\mathrm{b}}$ Muzaffar H. Faroqui, FFARCSI, ${ }^{\mathrm{c}}$ and \\ Robert S. Bonser, FRCS, MRCP, ${ }^{a}$ Birmingham, United Kingdom
}

Retrograde cerebral perfusion ( $\mathrm{RCP}$ ) through the superior vena cava (SVC) has been used to improve cerebral protection during hypothermic circulatory arrest (HCA) in aortic operations. Different mechanisms of protection have been postulated for RCP. These include the optimization of cerebral metabolic function during HCA by oxygenation and removal of catabolic products, maintaining a low brain temperature, and flushing of gaseous and particulate microemboli from the arterial tree before reinstitution of antegrade perfusion. Some of these hypotheses are based on the assumption that blood retrogradely perfused through the SVC actually reaches the

From the Cardiothoracic Surgical Unit, ${ }^{\text {a }}$ and the Departments of Nuclear Medicine ${ }^{\mathrm{b}}$ and Anaesthesia and Intensive Care, Queen Elizabeth Hospital, Birmingham, United Kingdom.

Accepted for publication June 26, 1995.

J Thorac CARdiovasc Surg 1996;111:270-2

Copyright (C) 1996 by Mosby-Year Book, Inc.

$0022-5223 / 96 \$ 5.00+0 \quad \mathbf{1 2 / 5 4 / 6 7 3 9 0}$ cerebral tissue. Although some evidence of this has been obtained in experimental studies, ${ }^{1}$ there has been no demonstration to date that this is the case in human beings. We report three cases in which cerebral perfusion during RCP was demonstrated with the technetium Tc $99 \mathrm{~m}$-labeled brain perfusion agent $\mathrm{d}, \mathrm{L}-$ hexamethyl propylene amine oxime ( ${ }^{99 \mathrm{~m}} \mathrm{Tc}-\mathrm{HMPAO}$; Amersham International Ltd., Little Chalfont, United Kingdom), and a portable gamma camera in the operating theater. ${ }^{99^{\mathrm{m}} \mathrm{Tc}-}$ HMPAO is a lipophilic compound that freely diffuses across the blood-brain barrier. Within the brain, it is either bound by a change in lipophilicity or by binding to intracellular components. This agent is useful for perfusion imaging because it is deposited in proportion to blood flow during the first pass through the cerebral circulation and has a very slow washout rate, ${ }^{2}$ thus acting as a "chemical microsphere." The protocol was approved by the local hospital ethics committee and by the Administration of Radioactive Substances Advisory Committee.

Three male patients (mean age 55 years) underwent aortic root and arch replacement for chronic aneurysmal disease.

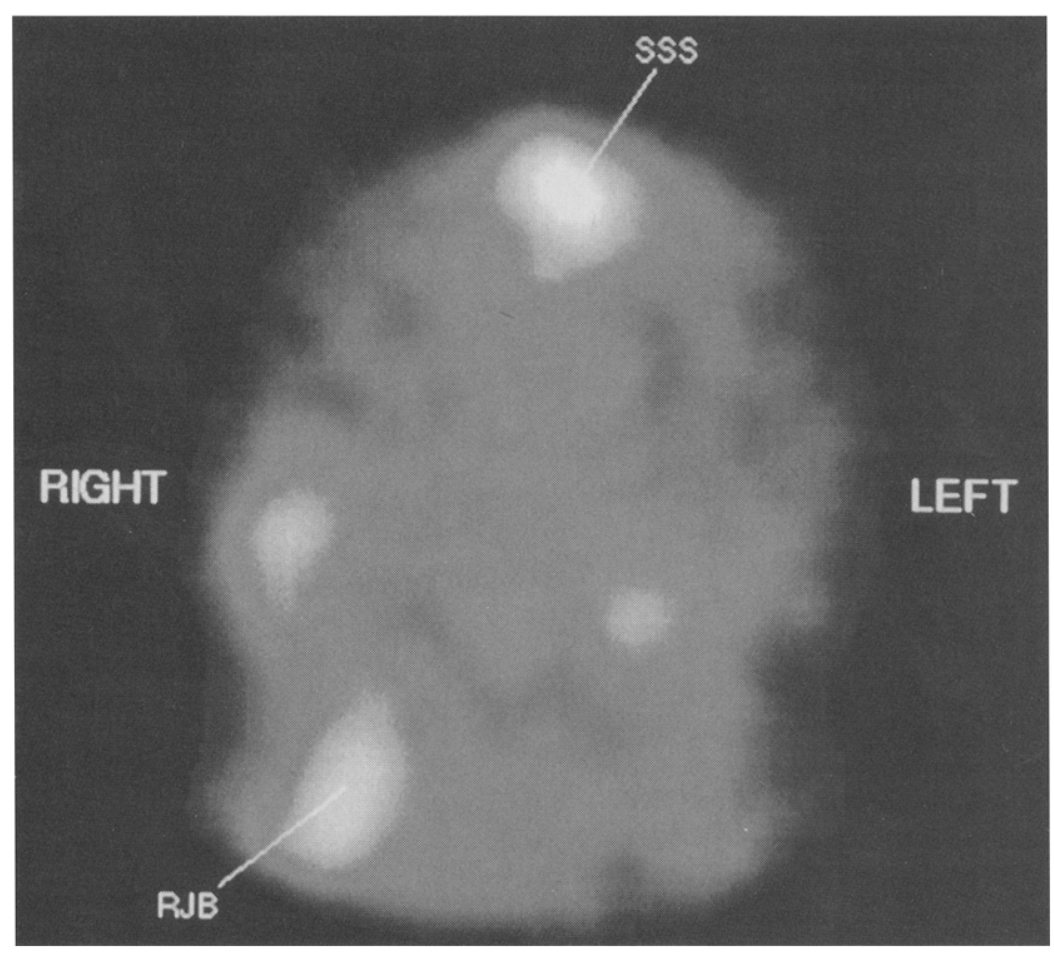

Fig. 1. Anterior image. Six minutes after commencement of RCP ${ }^{99 \mathrm{~m}} \mathrm{Tc}-\mathrm{HMPAO}$ activity can be seen predominately in the right jugular bulb $(R J B)$ and superior sagittal sinus (SSS). 


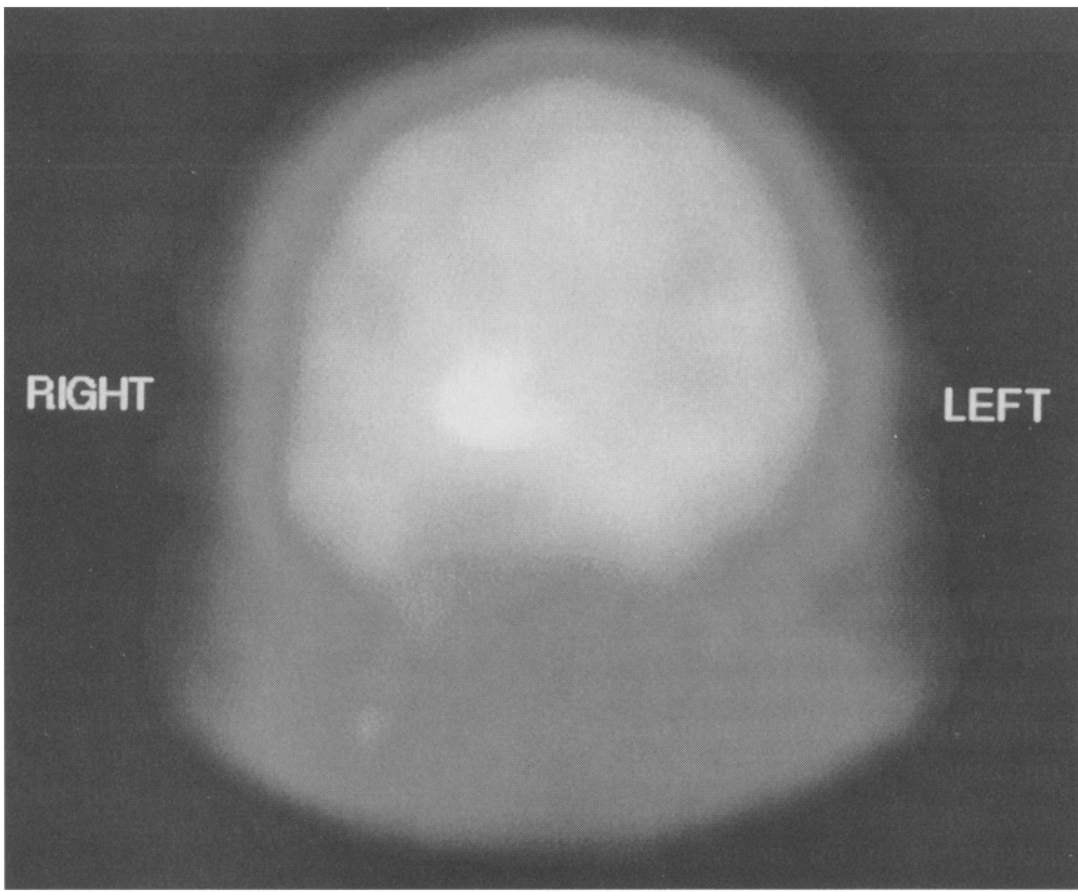

Fig. 2. Ten minutes after commencement of RCP, accumulation of ${ }^{99 n} \mathrm{Tc}-\mathrm{HMPAO}$ activity can be seen throughout the white and gray matter with reduced activity in the superior sagittal sinus, indicating accumulation of the agent in the brain and clearance from the vascular structures.

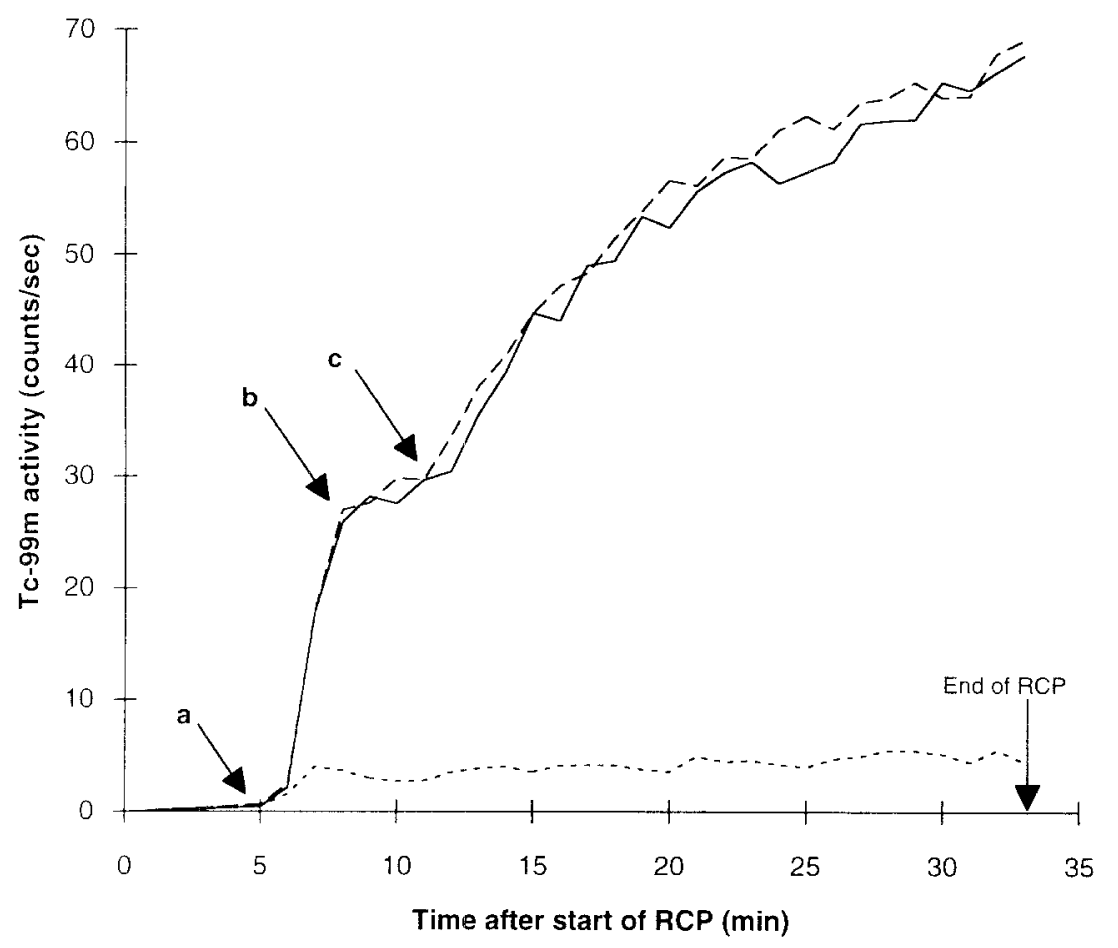

Fig. 3. Time activity curves constructed for the right and left cerebral hemispheres and a background region in the neck. The arrows show arrival of ${ }^{99 \mathrm{~m}} \mathrm{Tc}-\mathrm{HMPAO}$ bolus (a), completion of first pass of bolus (b), and attainment of complete mixing within the circulating blood volume (c). Thereafter the tracer continues to accumulate in the brain, with negative exponential uptake. 
Cardiopulmonary bypass was established by bicaval cannulation and common femoral arterial return. A parallel 0.25 inch cannula was connected between the arterial return and the SVC cannula by means of Y connectors to provide RCP after HCA. This was primed and clamped at both ends. After implementation of $\mathrm{HCA}$ (nasopharyngeal temperature $15^{\circ} \mathrm{C}$ ), the femoral arterial cannula was clamped and the blood was drained into the cardiotomy reservoir. The SVC cannula was isolated from the venous circulation, and RCP commenced with flow (mean $383 \pm 35 \mathrm{ml} / \mathrm{min}$ ) adjusted to maintain a right jugular bulb pressure of $25 \mathrm{~mm} \mathrm{Hg}$ or less. A dedicated retrograde right internal jugular line independent of any drug infusions was used to measure jugular bulb pressure. A detailed description of our RCP circuit can be found elsewhere. ${ }^{3}$

Before circulatory arrest, a portable gamma camera (Siemens LEM+; Siemens AG, Erlangen, Germany) linked to a dedicated nuclear medicine computer system (Gamma 11, Nuclear Diagnostics, Kent, United Kingdom) was placed anterior to the head of the patient. After HCA and immediately before the commencement of RCP, $100 \mathrm{MBq}$ of ${ }^{99 \mathrm{~m}} \mathrm{Tc}-\mathrm{HMPAO}$ was administered into the cardiotomy reservoir and allowed to diffuse. Planar dynamic brain imaging was commenced when the first sign of activity was seen in the SVC. Images were acquired every 2 seconds for the first 2 minutes and every minute thereafter for the duration of RCP (mean $32 \pm 7$ minutes). Inspection of the gamma camera images showed ${ }^{99 \mathrm{~m}}$ Tc-HMPAO activity spreading quickly from the right jugular bulb through the cerebral vascular tree (Fig. 1) and throughout the brain within 3 minutes (Fig. 2). Continuous ${ }^{99 \mathrm{~m}} \mathrm{Tc}-\mathrm{HMPAO}$ accumulation was seen in the gray and white matter for the duration of RCP, with reduction of activity in the superior sagittal sinus as the agent was cleared from the blood pool. Time activity curves constructed for both cerebral hemispheres and a background region in the neck demonstrate homogeneous perfusion in both cerebral hemispheres (Fig. 3).

One of the patients, who had previously undergone a repeated aortic valve replacement and coronary artery revascularization and had dissecting type $\mathrm{A}$ aortic aneurysm and vein graft occlusion, died of myocardial failure in the early postoperative period. Assessment of neurologic function was not possible because the patient was electively sedated during the postoperative course. The remaining two patients had normal return to consciousness in less then 24 hours after operation and had no neurologic complications.

This pilot study demonstrates that cerebral perfusion does occur with RCP in human beings. The question of whether the metabolic demands of the brain may be met with this technique remains to be answered.

\section{REFERENCES}

1. Usui A, Hotta T, Hiroura M, et al. Retrograde cerebral perfusion through a superior vena caval cannula protects the brain. Ann Thorac Surg 1992;53:47-53.

2. Costa DC, Ell PJ, Cullum ID, Jorritt PH. The in vivo distribution of ${ }^{99} \mathrm{Te}^{\mathrm{m}}-\mathrm{HM}-\mathrm{PAO}$ in normal man. Nucl Med Commun 1986;7:647-58.

3. Pagano D, Carey JA, Patel RL, et al. Retrograde cerebral perfusion: clinical experience in emergency and elective aortic operations. Ann Thorac Surg 1995;59:393-7.

\title{
RETRANSFUSION OF THORACIC WOUND BLOOD DURING HEART SURGERY OBSCURES BIOCOMPATIBILITY OF THE EXTRACORPOREAL CIRCUIT
}

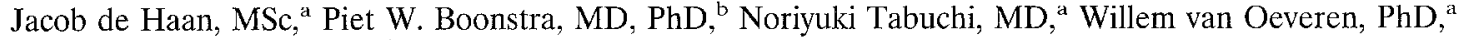
and Tjark Ebels, $\mathrm{MD}, \mathrm{PhD},{ }^{\mathrm{b}}$ Groningen, The Netherlands

To minimize blood trauma and postoperative pathologic outcomes, it is of great concern to improve the biocompatibility of extracorporeal circuits.' Worldwide, many comparative studies have been performed to select the most biocompatible extracorporeal circuit. In many of these studies, however, a systematic error was made.

From the Blood Interaction Research Division ${ }^{a}$ and the Department of Cardiothoracic Surgery, University Hospital Groningen, The Netherlands.

Accepted for publication May 31, 1995.

J Thorac Cardiovasc Surg 1996;111:272-5

Copyright $(\mathcal{C} 1996$ by Mosby-Year Book, Inc.

$0022-5223 / 96 \$ 5.00+0 \quad \mathbf{1 2 / 5 4 / 6 6 6 0 4}$
Retransfusion of heparinized blood from the wound area is an established method to attenuate excessive blood loss during cardiopulmonary bypass (CPB). Retransfusion of this wound blood is feasible by suction from the thoracic cavity and recirculation through the cardiotomy reservoir. Because wound blood is highly traumatized, as we demonstrated in recent studies, ${ }^{2,3}$ there are likely to be significant effects of wound blood retransfusion on the systemic blood, depending on the amount of retransfused wound blood. Particularly, retransfusion of the damaged wound blood could obscure possible improvements in biocompatibility of extracorporeal circuits. ${ }^{4}$ In this study, wound blood was retained during CPB in one group of 14 patients and retransfused only at the end of bypass. The control group consisted of 20 other patients, who were 